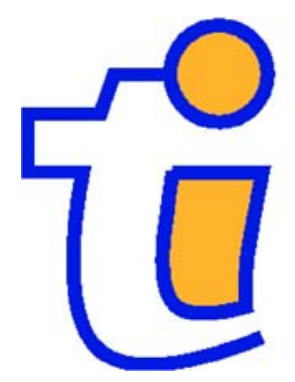

TI 2004-102/2

Tinbergen Institute Discussion Paper

Weighted Sums of Subexponential Random Variables and Asymptotic Dependence between Returns on Reinsurance Equities

\author{
J.L. Geluk \\ C.G. de Vries*
}

Faculty of Economics, Erasmus Universiteit Rotterdam, and Eurandom, Eindhoven.

* Tinbergen Institute. 
Tinbergen Institute

The Tinbergen Institute is the institute for economic research of the Erasmus Universiteit Rotterdam, Universiteit van Amsterdam, and Vrije Universiteit Amsterdam.

Tinbergen Institute Amsterdam

Roetersstraat 31

1018 WB Amsterdam

The Netherlands

Tel.: $\quad+31(0) 205513500$

Fax: $\quad+31(0) 205513555$

Tinbergen Institute Rotterdam

Burg. Oudlaan 50

3062 PA Amsterdam

The Netherlands

Tel.: $\quad+31(0) 104088900$

Fax: $\quad+31(0) 104089031$

Please send questions and/or remarks of nonscientific nature to driessen@tinbergen.nl.

Most TI discussion papers can be downloaded at http://www.tinbergen.nl. 


\title{
WEIGHTED SUMS OF SUBEXPONENTIAL RANDOM VARIABLES AND ASYMPTOTIC DEPENDENCE BETWEEN RETURNS ON REINSURANCE EQUITIES
}

\author{
J.L. GELUK AND C.G. DE VRIES \\ Erasmus University Rotterdam \\ P.O. Box 1738, 3000 DR Rotterdam, The Netherlands \\ Eurandom, P.O. Box 513, 5600 MB Eindhoven, The Netherlands
}

\begin{abstract}
Suppose $X_{1}, X_{2}, \ldots$ are independent subexponential random variables with partial sums $S_{n}$. We show that if the pairwise sums of the $X_{i}$ 's are subexponential, then $S_{n}$ is subexponential and $P\left(S_{n}>\right.$ $x) \sim \sum_{1}^{n} P\left(X_{i}>x\right)(x \rightarrow \infty)$. The result is applied to give conditions under which $P\left(\sum_{1}^{\infty} c_{i} X_{i}>x\right) \sim \sum_{1}^{\infty} P\left(c_{i} X_{i}>x\right)$ as $x \rightarrow \infty$, where $c_{1}, c_{2}, \ldots$ are constants such that $\sum_{1}^{\infty} c_{i} X_{i}$ is a.s. convergent. Asymptotic tail probabilities for bivariate linear combinations of subexponential random variables are given. These results are applied to explain the joint movements of the stocks of reinsurers. Portfolio investment and retrocession practices in the reinsurance industry, for reasons of diversification, exposes different reinsurers to the same risks on both sides of their balance sheets. Assuming, in line with the industry practice that the risk drivers follow subexponential distributions, we derive (under mild conditions) when the reinsurer's equity returns are asymptotically dependent, exposing the industry to systemic risk.
\end{abstract}

AMS 1991 subject classification. Primary 60F05; secondary 62E20.

Key words. Subexponentiality, regular variation, systemic risk, asymptotic dependence.

Date: 12th September 2004. 


\section{INTRODUCTION AND MAIN RESULT}

The spate of very large insurance claims coupled with the recent creeping stock market decline has eroded the financial wealth of many reinsurance companies. In this paper we provide probabilistic arguments as to why the reinsurance industry as a whole fared so badly. The total returns on the assets and liabilities, and hence the equity, of reinsurers can be written as a weighted sum of returns on the individual exposures and investments. To investigate the interdependencies between different reinsurance companies we therefore investigate the joint behavior of different weighted sums of random variables which represent the returns on individual exposures (positive or negative). This joint behavior determines the exposure of the reinsurance industry to systemic risk. In line with much of the empirical evidence for the non-life insurance business, we focus on the joint behavior of the individual risk drivers with heavy tailed (subexponential) tail behavior.

The asymptotic behavior of the tail of $Z=\sum c_{i} X_{i}$ for i.i.d. random variables $X_{i}(i=1,2, \ldots)$ with d.f. $F$ has received considerable attention over time. The case of the normal distribution is well known and has a long history dating back to the start of the field. Early results for the heavy tailed situation use regular variation. In case $P\left(\left|X_{i}\right|>x\right)$ is regularly varying with index $-\alpha(\alpha>0)$, typical results are given in the form

$$
\lim _{x \rightarrow \infty} \frac{P(|Z|>x)}{P\left(\left|X_{i}\right|>x\right)}=\sum\left|c_{i}\right|^{\alpha}
$$

under different conditions on the coefficients. The first result in this direction in case $\alpha \leq 1$ is given by Cline [5]. See also Resnick [35], Lemma 4.24 for a proof which does not use Tauberian results. His result was subsequently extended by Kokoszka and Taqqu ([24], Theorem 2.2) to the case $1<\alpha<2$. Recently Mikosch and Samorodnitsky [30] used conditions on the coefficients which are close to best possible

$$
\begin{aligned}
& \sum c_{i}^{2}<\infty \text { for } \alpha>2, \\
& \sum\left|c_{i}\right|^{\alpha-\varepsilon}<\infty, \text { for some } \varepsilon>0 \text { for } \alpha \leq 2
\end{aligned}
$$

and assumed that, if $\alpha>1, E X=0$. In that case under the additional tail balance condition

$$
\bar{F}(x) \sim p P\left(\left|X_{i}\right|>x\right), F(-x) \sim q P\left(\left|X_{i}\right|>x\right), x \rightarrow \infty,
$$

where $p \in(0,1], q=1-p$, it follows that as $x \rightarrow \infty$

$$
\frac{P(Z>x)}{P\left(\left|X_{i}\right|>x\right)} \rightarrow \sum_{i}\left|c_{i}\right|^{\alpha}\left[p I_{c_{i}>0}+q I_{c_{i}<0}\right] \text {. }
$$


In contrast to the situation with a regularly varying tail, the asymptotic behaviour of the tail of $Z$ in the situation where the innovations have a balanced and rapidly varying tail depends only on the coefficients $c_{i}$ for which $\left|c_{i}\right|$ is maximal. In particular, if $F$ is subexponential and in the domain of attraction of the Gumbel distribution, Davis and Resnick [9] show that under the conditions

$$
\sum\left|c_{i}\right|^{\delta}<\infty \text { for some } \delta \in(0,1) \text { and } \max _{i}\left|c_{i}\right|=1,
$$

and the tail balance condition (1.2), that

$$
P(Z>x) \sim\left(p k^{+}+q k^{-}\right) P(|X|>x)(x \rightarrow \infty),
$$

where $k^{+}=\operatorname{card}\left\{i: c_{i}=1\right\}, k^{-}=\operatorname{card}\left\{i: c_{i}=-1\right\}$.

In this paper we give a general formulation of the above mentioned results for the class of random variables with a subexponential distribution. Let $X_{1}, X_{2}$ be independent and identically distributed random variables with distribution function $F$ with $F(x)<1$ for all $x$. The distribution is subexponential $(F \in \mathscr{S})$ if

$$
P\left(X_{1}+X_{2}>x\right) \sim 2 P\left(X_{1}>x\right) \text { as } x \rightarrow \infty .
$$

The theory of subexponential distributions is by now well established and its relevance is obvious from applications in various areas of applied probability. For a recent review of applications of subexponentiality in different areas, the reader is referred to the book by Embrechts, Klüppelberg and Mikosch [13] or, more recently, Asmussen [1].

The class $\mathscr{S}$ is related to several other classes of functions. A well known result is the inclusion $\mathscr{S} \subset \mathscr{L}$, where $\mathscr{L}$ is the class of long tailed distribution functions $F$ satisfying $\bar{F}(x+a) / \bar{F}(x) \rightarrow 1$ as $x \rightarrow \infty$ (for $a \in \mathbf{R}$ ), where $\bar{F}(x):=P(X>x)$. In this case convergence is uniform on compact subsets of $\mathbf{R}$. We will also use the notation $\mathscr{L}$ for functions $\phi$ satisfying $\phi(x+a) \sim \phi(x)$.

There is a connection with functions of dominated variation as well: the inclusion $\mathscr{L} \cap \mathscr{D} \subset \mathscr{S}$ (see [18]) holds. We write $F \in \mathscr{D}$ to denote that the tail function $\bar{F}$ is of dominated variation, i.e. if $\limsup _{x \rightarrow \infty} \bar{F}(a x) / \bar{F}(x)<$ $\infty$ for $a<1$.

It follows from the above inclusion that the class $\mathscr{R} \mathscr{V}$ of distributions with a regularly varying tail function $\bar{F}$ is a subset of $\mathscr{S}$. A different proof of this inclusion is given in Feller [15]. Apart from distributions with a regularly varying tail like the Pareto, Student's $t$, the loggamma and the $F$ distribution, other examples of subexponential distributions include the Weibull distribution $\bar{F}(x)=c \exp \left(-x^{\alpha}\right)$ with $\alpha<1$ and the lognormal 
distribution. Note that it is no restriction to set the scale parameter to be 1 (see the beginning of section 2).

It is well-known that the sum of two independent, not identically distributed subexponential random variables is not necessarily subexponential. See ([25]) for a counterexample. We prove (see Theorem 3 below) that if pairwise sums of independent random variables have a subexponential distribution, then the partial sums have a subexponential distribution. This result is a tool in proving our main result, Theorem 1 below, which extends the above mentioned results on weighted sums of regularly varying random variables. It turns out that neither the regular (rapid) variation nor tail balance assumption is essential in order to obtain the result. The assumption of regular variation of the tail function is replaced with subexponentiality plus a one-sided growth condition. Although the assumption of balanced tails is weakened significantly, it is nevertheless possible to give an asymptotic estimate of the right tail $P(Z>x)$ separately. Because of the weak assumptions on tail balance, we need to make separate assumptions on the left-hand tail.

It should be observed that some additional assumptions like (1.5) below and $b 1-b 3$ are necessary. This follows from Leslie's result [25]: take $X_{1}, X_{2}$ i.i.d. random variables such that the d.f. of $X_{1}$ and $-X_{2}$ are subexponential, but $X_{1}-X_{2}$ does not have a subexponential distribution function. Lemma 5 below shows that $X_{1}$ is not max-sum equivalent with $-X_{2}$, hence $\lim _{x \rightarrow \infty} P\left(X_{1}-X_{2}>x\right) /\left(P\left(X_{1}>x\right)+P\left(-X_{2}>x\right)\right) \neq 1$ and (1.6) below does not hold.

In order to study the asymptotic behaviour of the tail of $Z$ under the assumption of subexponentiality of the underlying random variables, we need to extend the definition of $\mathscr{S}$ to the real line. The defining relation is again (1.4). Subexponential distributions on the real line were studied before by Willekens [40], Omey [31] and Geluk [16].

We use the notation $a \vee b=\max (a, b), a \wedge b=\min (a, b)$ and in case $X, Y$ are independent random variables with d.f. $F, G$, we write $F * G$ for the d.f. of the convolution $X+Y$ and $\overline{F * G}(x)=P(X+Y>x)$. Moreover we write $f(x) \asymp g(x)$ to denote $f(x)=O(g(x))$ and $g(x)=O(f(x))$ as $x \rightarrow \infty$.

For a random variable $X$ with d.f. $F$, we use the notation $F^{+}\left(F^{-}\right)$for the distribution function of the random variable $X^{+}:=X \vee 0\left(X^{-}:=\right.$ $-(X \wedge 0))$. Unless mentioned otherwise $F(G)$ denotes the d.f. of the random variable $X(Y)$. 
Theorem 1. Suppose $X, X_{1}, X_{2}, \ldots$ are i.i.d. random variables with d.f. $F \in \mathscr{S}$ such that there exist $\alpha, \beta$ with

$$
\lambda^{-\beta} \leq \liminf _{x \rightarrow \infty} \frac{P(|X|>\lambda x)}{P(|X|>x)} \leq \limsup _{x \rightarrow \infty} \frac{P(|X|>\lambda x)}{P(|X|>x)} \leq \lambda^{-\alpha} \text { for } 0<\lambda \leq 1 .
$$

Assume that $\left(c_{i}\right)_{i \geq 1}$ is a sequence such that $c_{i}>0$ for some $i \geq 1$ and

$$
\begin{aligned}
& \sum c_{i}^{2}<\infty \text { for } \beta>2, \\
& \sum\left|c_{i}\right|^{\beta}<\infty \text { for } \beta \leq 2 .
\end{aligned}
$$

Moreover assume that the left-hand tail d.f. $F^{-}$satisfies one of the conditions

b1 $F^{-} \in \mathscr{L}$ and $P(X<-c x)=O(P(X>x))(x \rightarrow \infty)$ for all $c>0$.

b2 $P(X<-c x)=o(P(X>x))(x \rightarrow \infty)$ for all $c>0$.

b3 $F^{-} \in \mathscr{L}$ and $P(X>x) \asymp P(X<-x)$ as $x \rightarrow \infty$.

Then as $x \rightarrow \infty$

$$
P\left(\sum_{i=1}^{\infty} c_{i} X_{i}>x\right) \sim \sum_{i=1}^{\infty} P\left(c_{i} X_{i}>x\right) .
$$

Note that the assumptions of the theorem imply that not only $F$ is subexponential, but the distribution function of $|X|$ is subexponential as well, being of extended regular variation. See e.g. Cline and Hsing [7] or Rolski et al. [36].

Observe that if $P(|X|>x)$ is regularly varying with exponent $-\alpha$ and the tail balance condition (1.2) holds, the assumptions of the theorem are satisfied and the above mentioned result by Mikosch and Samorodnitsky [30] follows from theorem 1.

In order to give the proof of Theorem 1, a closure result on sums of independent subexponential random variables is used (Theorem 3 ). The theorem extends earlier results on the tail probability of sums. In section 3 the proof of theorem 1 is given.

In sections 4 and 5 we show how the technique developed in previous sections can be applied to insurance economics. Reinsurance companies reinsure parts of the insurance contracts written by insurance companies and also reinsure other reinsurance contracts, which is the business of retrocession. In the proportional treaty reinsurance business, like the popular quota-share contract, the reinsurer shares proportionally in the premiums and the claims of the ceding company. It follows that different reinsurance companies hold different proportions of the same underlying risks. The motive for spreading the risk over multiple companies is the diversification 
of risk, i.e. the exploitation of the law of large numbers (assuming that the first moment exists; note that most insurance and reinsurance treaties are capped). On the asset side of the balance sheet of reinsurance companies one usually finds large diversified portfolios of bond and equity investments. Again, the motivation for holding a diversified portfolio is to reduce the risk. This practice implies that many reinsurance companies hold stakes in the same companies. Under the proportional treaty business, portfolios of reinsurance treaties are by nature linear in the underlying risks; investment portfolios are by nature linear in the invested assets. Thus the asset and liability sides of the reinsurer's balance sheet can be represented as linear combinations of underlying risks.

In the empirical finance literature it is now generally accepted that sharp asset price fluctuations tend to occur more often than predicted on the basis of the popular normal distribution. Since the seminal work by Mandelbrot [29], numerous studies have estimated heavy tailed distribution functions like the stable, Student-t, and other subexponential distributions. The relative occurrence of stock market extremes has by far received the most attention, see e.g. Blattberg and Gonedes [3], Jansen and de Vries [21], Lux [28], Longin [26] or Jondeau and Rockinger [22]; bond market extremes have been considered in de Haan et al. [19].

Similarly, in the empirical literature on non-life insurance claims, the heavy tail feature has long been recognized. See for example, Pfeifer [32], Embrechts et al.[13] and Reiss and Thomas [34]. This empirical fact has been integrated with ruin theory and has changed the classical Cramer theory for insurance premia. This is well documented in Embrechts et al. [13].

In this paper we consider the class of subexponential distributions to capture the heavy tail feature. Assuming that the distributions of the underlying risk drivers are subexponential is common for non-life insurance claims, and fits nicely to the empirical observation of heavier tails than the normal. The same observation applies to equity returns. Combine this feature with the linearity of the portfolios of reinsurers balance sheets. Given that both investment returns as well as the claims are subexponential distributed, we investigate when gross returns of the (re)insurance companies are subexponentially distributed. The additivity property foolows from the linearity of the portfolios combined with some natural (economic) conditions. Subsequently we investigate the implications for the entire industry. It is shown that the (mis)fortunes of different reinsurance companies become asymptotically dependent. Only if reinsurance companies would hold non-diversified unique investment portfolios and reinsurance treaties, so that each reinsurer holds exposures that are not held by others, the asymptotic dependence disappears. The sector is therefore subject to systemic risk. In effect, one might loosely say that for the extreme risks, the sector acts as a single company. 
The flip side is that for the smaller risks, the diversification is beneficial, since there the industry will experience less solvency problems due to the smearing out of these risks across the sector. Together these facts and features explain why the reinsurance industry as a whole was badly affected by the 2001-2003 stock market decline.

The above implies that the equity returns of different reinsurance companies should exhibit dependence even in extreme cases. We provide some graphical evidence for this reduced form implication.

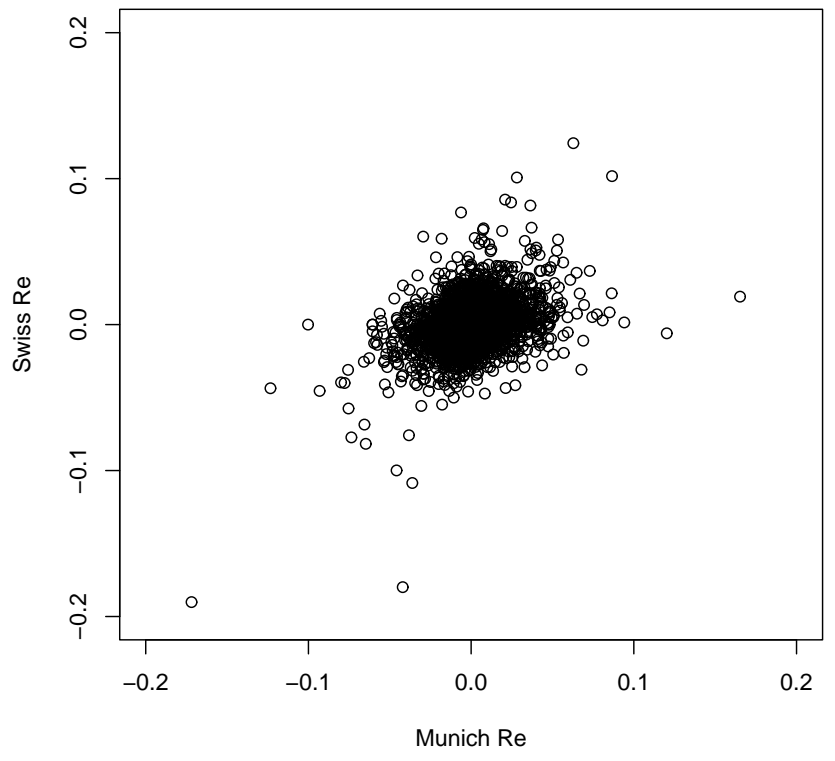

FIGURE 1. Daily stock returns of Munich Re and Swiss Re.

The crossplot in Figure 1 contains the daily logarithmic stock return of the two top reinsurance companies Munich RE and Swiss RE over the period 1990-2002. The returns of the two reinsurers are clearly not independent, the correlation coefficient is $r=0.371$. One notices a number of outliers, which sometimes appear to occur jointly.

To put this crossplot into perspective, we take the estimated correlation coefficient together with the means and variances of the two marginal distributions and generate an equal amount of bivariate normally distributed pseudo random numbers. The sample is shown in the next figure using the same scale. 


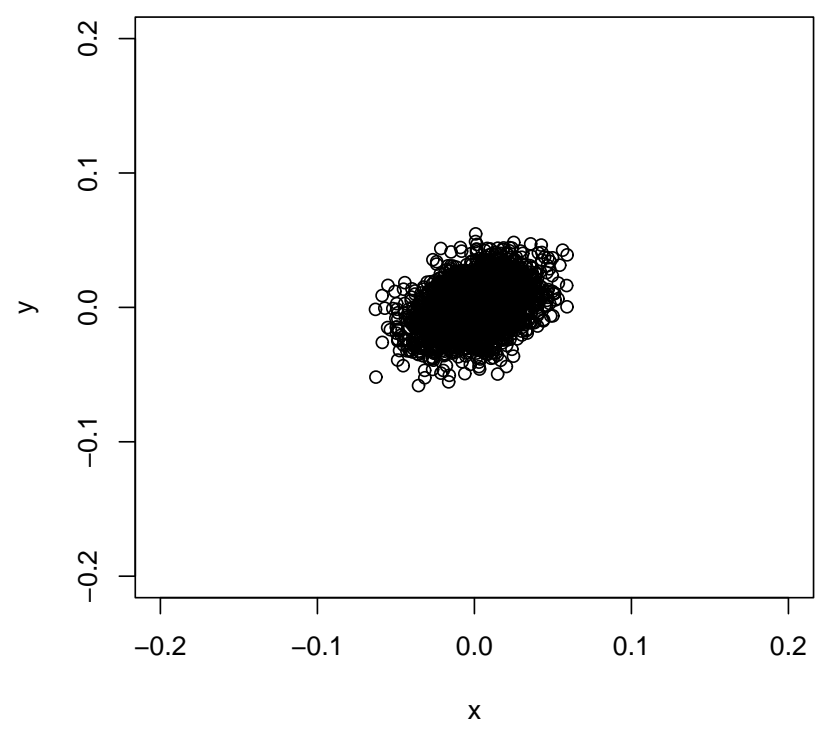

FIGURE 2. A sample from the bivariate normal distribution.

The plots differ markedly in the extreme North-East and South-West corners. The true data contain many more outliers, which moreover are repeatedly located closer to the diagonal than the axes, and thus appear to occur jointly. The correlation cum normal assumption does not adequately capture the dependency in the tail areas (of the distribution) observed in the first crossplot.

The observed discrepancy between the two figures is not atypical for asset markets in general. The empirical literature has concluded the concept of normal based correlation does not adequately capture the dependency structure. See e.g. Longin and Solnik [27], Hartmann et al. [20] and Poon et al. [33].

The fat tail feature reveals that extreme returns are more likely than one would expect in a normally distributed world. However, in order to assess the extremal effect on portfolios and global financial stability one also needs to evaluate the potential for big collapses in nominal asset values to strike simultaneously. A recent strand in the spillover literature circumvents the use of correlations and directly focuses on dependence structure of multivariate distributions in the tails by using multivariate statistical extreme value analysis. 
Analyses on foreign exchange data have recently been carried out by Starica [38] and for major stock markets by Straetmans [39], Longin and Solnik [27], Poon et al. [33] and Hartmann et al. [20]. The latter three papers find evidence for asset markets to be asymptotically dependent, but also identify cases where these markets are asymptotically independent.

Thus, fat tails and tail dependence for asset returns have by now been documented in the empirical finance and insurance literature. To the best of our knowledge, however, how the marginal tail thickness of the asset returns relates theoretically to the multivariate tail dependence between reinsurance asset return series has not been dealt with before.

\section{TAIL PROBABILITIES FOR WEIGHTED SUMS OF NON-NEGATIVE RANDOM VARIABLES}

Unless mentioned otherwise, the random variables in this section are a.s. non-negative.

A fundamental role in the proofs of our results is played by the notion of max-sum equivalence, which was introduced in Embrechts and Goldie ([11]).

Definition 1. $F_{1}$ and $F_{2}$ are said to be max-sum equivalent, written $F_{1} \sim_{M}$ $F_{2}$ if

$$
\overline{F_{1} * F_{2}}(x) \sim \bar{F}_{1}(x)+\bar{F}_{2}(x), x \rightarrow \infty .
$$

Note that $\bar{F}_{1}(x)+\bar{F}_{2}(x) \sim \bar{F}_{1}(x)+\bar{F}_{2}(x)-\bar{F}_{1}(x) \bar{F}_{2}(x)$, so we may rewrite $(2.1)$ as

$$
P\left(X_{1}+X_{2}>x\right) \sim P\left(X_{1} \vee X_{2}>x\right)(x \rightarrow \infty),
$$

which explains the terminology. Subexponentiality can be expressed in terms of the relation $\sim_{M}$ as well: $F \in \mathscr{S}$ if and only if $F \sim_{M} F$. Observe that relation (2.2) implies that for a r.v. $X$ with d.f. $F \in \mathscr{S}$ and $c>0$, the r.v. $c X$ has d.f. in $\mathscr{S}$.

The following result ([11], theorem 2) is fundamental:

Theorem 2. Let $X, Y$ be independent with d.f. $F$ and $G, F \in \mathscr{S}, G \in \mathscr{S}$ and $H=F * G$. Then the following are equivalent:

(1) $H \in \mathscr{S}$,

(2) $F \sim_{M} G$,

(3) $p F+(1-p) G \in \mathscr{S}$ for some (all) p satisfying $0<p<1$.

It is well known that for $X_{i}(1 \leq i \leq n)$ i.i.d. with d.f. $F \in \mathscr{S}$, relation (1.4) implies

$$
P\left(X_{1}+X_{2}+\ldots+X_{n}>x\right) \sim n P\left(X_{1}>x\right)(x \rightarrow \infty) .
$$


See Chistyakov [4]. Conversely the class $\mathscr{S}$ is closed under convolution roots: if (2.3) holds, then $F \in \mathscr{S}$. See Embrechts et al. ([10], thm. 2).

Our first result is an extension of (2.3) above to the case of independent variables.

Extending earlier results in Cline [6] and Embrechts and Goldie [12], the following result is given in [13]. If $X_{1}, X_{2}, \ldots, X_{n}$ are independent r.v.'s, $F$ is a d.f. in $\mathscr{S}$ and

$$
\lim _{x \rightarrow \infty} \frac{P\left(X_{i}>x\right)}{\bar{F}(x)}=a_{i} \in[0, \infty], i=1, \ldots, n,
$$

then

$$
\lim _{x \rightarrow \infty} \frac{P\left(\sum_{i=1}^{n} X_{i}>x\right)}{\bar{F}(x)}=\sum_{i=1}^{n} a_{i} .
$$

In the next theorem the assumption (2.4) of tails which have a (possibly infinite) limit as compared to the tail function $\bar{F}$ is replaced with the assumption (2.5) below. This assumption is more general in the sense that the limits in (2.4) are not supposed to exist.

Theorem 3. Let $X_{1}, X_{2}, \ldots$ be independent non-negative random variables with d.f's $F_{i}(i=1,2, \ldots)$. Suppose

$$
F_{i} * F_{j} \in \mathscr{S} \text { for } i, j \geq 1 \text {. }
$$

Then

$$
F_{i} \in \mathscr{S}(i \geq 1), \quad F_{1} * F_{2} \ldots * F_{n} \in \mathscr{S}
$$

and

$$
P\left(\sum_{i=1}^{n} X_{i}>x\right) \sim \sum_{i=1}^{n} P\left(X_{i}>x\right), x \rightarrow \infty .
$$

The next result is a consequence in the i.i.d. situation.

Corollary 1. Suppose the random variables $X_{k}(k=1,2, \ldots, n)$ are i.i.d. distributed with d.f. $F \in \mathscr{S}$. Suppose $S_{n}$ defined by

$$
S_{n}:=\sum_{i=1}^{n} c_{i} X_{i}
$$

has d.f. $K_{n}$. If $c_{i}>0(1 \leq i \leq n)$, then $K_{n} \in \mathscr{S}$ and

$$
P\left(\sum_{i=1}^{n} c_{i} X_{i}>x\right) \sim \sum_{i=1}^{n} P\left(c_{i} X_{i}>x\right), x \rightarrow \infty .
$$


Proof of Corollary 1. According to Theorem 3, it is sufficient to show that $c_{i} X_{i}+c_{j} X_{j}$ has a subexponential d.f. for $i, j \geq 1$. In case $c_{i} \leq c_{j}$, this follows from thm. 1 in [11] since $c_{j} X_{j}$ has a subexponential distribution function and $\sup _{x} P\left(c_{i} X_{i}>x\right) / P\left(c_{j} X_{j}>x\right) \leq 1<\infty$. In case $c_{j} \leq c_{i}$ a similar argument completes the proof.

For the proof of theorem 3 we need two lemmas.

Lemma 1. Suppose $Z_{1}, Z_{2}$ are independent non-negative r.v.'s with distribution functions $H_{1}, H_{2}$, satisfying $H_{1} \sim_{M} H_{2}$, where $H_{1} \in \mathscr{L}$.

If $\phi(x)=o\left(\bar{H}_{2}(x)\right)(x \rightarrow \infty)$ and $\phi$ is locally bounded, then

$$
\int_{0}^{x} \phi(x-u) d H_{1}(u)=o\left(\bar{H}_{1}(x)+\bar{H}_{2}(x)\right)(x \rightarrow \infty) .
$$

Proof of Lemma 1. Take $\varepsilon>0$ arbitrary. There exists $a>0$ such that $\phi(x) \leq \varepsilon \bar{H}_{2}(x)$ for $x>a$. Hence

$\left|\int_{0}^{x} \phi(x-u) d H_{1}(u)\right| \leq \varepsilon \int_{0}^{x-a} \bar{H}_{2}(x-u) d H_{1}(u)+M\left(\bar{H}_{1}(x-a)-\bar{H}_{1}(x)\right)$,

where $M>0$ is a constant. Note that, since $H_{1} \in \mathscr{L}$, it follows that for $x$ sufficiently large

$$
\bar{H}_{1}(x-a)-\bar{H}_{1}(x) \leq \varepsilon \bar{H}_{1}(x) .
$$

Since $H_{1} \sim_{M} H_{2}$, we have

$$
\begin{aligned}
P\left(Z_{1}+Z_{2}>x\right)-P\left(Z_{1} \vee Z_{2}>x\right) & =\int_{0}^{x}\left(\bar{H}_{2}(x-u)-\bar{H}_{2}(x)\right) d H_{1}(u) \\
& =o\left(\bar{H}_{1}(x)+\bar{H}_{2}(x)\right),
\end{aligned}
$$

hence

$$
\int_{0}^{x} \bar{H}_{2}(x-u) d H_{1}(u)=\bar{H}_{2}(x)+o\left(\bar{H}_{1}(x)+\bar{H}_{2}(x)\right) .
$$

Since $\varepsilon>0$ is arbitrary, the statement of the lemma follows from (2.9)(2.11).

We use the notation $S_{k}:=X_{1}+\ldots+X_{k}, M_{k}:=X_{1} \vee \ldots \vee X_{k}$ and $G_{k}:=P\left(M_{k} \leq x\right)(1 \leq k \leq n)$.

Lemma 2. Under the assumptions of theorem 3, we have $G_{n-1} \sim_{M} F_{n}(n \geq$ 2).

Proof of Lemma 2. Note that for constants $a_{i}, b_{i}$ satisfying $0<b_{i}<a_{i}<$ $1, i=1,2, \ldots n$, we have

$$
\prod_{i=1}^{n-1} a_{i}-\prod_{i=1}^{n-1} b_{i} \leq \sum_{i=1}^{n-1}\left(a_{i}-b_{i}\right) .
$$


By theorem 2 we have $F_{i} \sim_{M} F_{j}$, whence

$$
\begin{aligned}
0 \leq \int_{0}^{x} & \left(F_{i}(x)-F_{i}(x-u)\right) d F_{j}(u)=P\left(X_{i}+X_{j}>x\right)-P\left(X_{i} \vee X_{j}>x\right) \\
& =o\left(\bar{F}_{i}(x)+\bar{F}_{j}(x)\right) .
\end{aligned}
$$

Now take $a_{i}=F_{i}(x)$ and $b_{i}=F_{i}(x-u)$ in (2.12) and integrate in order to obtain

$$
\int_{0}^{x} \prod_{i=1}^{n-1} F_{i}(x-u) d F_{n}(u)=\prod_{i=1}^{n} F_{i}(x)+o\left(\sum_{i=1}^{n} \bar{F}_{i}(x)\right),(x \rightarrow \infty) .
$$

In order to prove the Lemma, we have to show that $G_{n-1} \sim_{M} F_{n}$, that is

$$
P\left(M_{n-1}+X_{n}>x\right) \sim P\left(M_{n-1} \vee X_{n}>x\right)=P\left(M_{n}>x\right) .
$$

This follows from (2.13) since

$$
P\left(M_{n-1}+X_{n}>x\right)=1-\int_{0}^{x} \prod_{i=1}^{n-1} F_{i}(x-u) d F_{n}(u) .
$$

and

$$
P\left(M_{n}>x\right)=1-\prod_{i=1}^{n}\left(1-\bar{F}_{i}(x)\right) \sim \sum_{i=1}^{n} \bar{F}_{i}(x)(x \rightarrow \infty) .
$$

Proof of Theorem 3. Note that $F_{i} \in \mathscr{S}(1 \leq i \leq n)$ follows from (2.5) (with $i=j$ ), since the class $\mathscr{S}$ is closed under convolution roots by theorem 2 in Embrechts et al. [10].

We now prove (2.7). Observe that in view of (2.15) and

$$
P\left(S_{n}>x\right)=P\left(M_{n}>x\right)+P\left(S_{n}>x, M_{n} \leq x\right),
$$

it is sufficient to prove that

$$
P\left(S_{n}>x, M_{n} \leq x\right)=o\left(P\left(M_{n}>x\right)\right), x \rightarrow \infty .
$$

The proof of (2.16) is by induction. Write

$$
P\left(S_{n}>x, M_{n} \leq x\right)=: I+J,
$$

where

$$
I=\int_{0}^{x} P\left(S_{n-1}>x-u, M_{n-1} \leq x-u\right) d F_{n}(u)
$$

and

$$
J=\int_{0}^{x} P\left(S_{n-1}>x-u, x-u<M_{n-1} \leq x\right) d F_{n}(u) .
$$

By Lemma 2 we have $G_{n-1} \sim_{M} F_{n}$, so we may apply Lemma 1 (take $\left.\phi(x)=P\left(S_{n-1}>x, M_{n-1} \leq x\right), H_{1}=F_{n}, H_{2}=G_{n-1}\right)$. Since $F_{n} \in$ $\mathscr{S} \subset \mathscr{L}$ the induction hypothesis and Lemma 1 imply $I=o\left(\bar{F}_{n}(x)+\right.$ 
$\left.\bar{G}_{n-1}(x)\right)$. In view of (2.15) this means $I=o\left(P\left(M_{n}>x\right)\right), x \rightarrow \infty$. Note that

$$
\begin{aligned}
J & \leq \int_{0}^{x} P\left(x-u<M_{n-1} \leq x\right) d F_{n}(u) \\
& =\int_{0}^{x}\left[P\left(M_{n-1} \leq x\right)-P\left(M_{n-1} \leq x-u\right)\right] d F_{n}(u) .
\end{aligned}
$$

Application of (2.13) completes the proof of (2.7).

In order to prove the second statement in (2.6), we use an induction argument: by assumption $F_{1} * F_{2} \in \mathscr{S}$. Suppose $F_{1} * F_{2} \ldots * F_{k} \in \mathscr{S}$ for some $k \in\{2,3, \ldots, n-1\}$.

Note that $P\left(S_{k} \vee X_{k+1}>x\right) \sim P\left(S_{k}>x\right)+P\left(X_{k+1}>x\right)$. Applying (2.7), it follows that $P\left(S_{k+1}>x\right) \sim P\left(S_{k}>x\right)+P\left(X_{k+1}>x\right)$. Combination gives $P\left(S_{k}+X_{k+1}>x\right) \sim P\left(S_{k} \vee X_{k+1}>x\right)$. Using $F_{k+1} \in \mathscr{S}$ and the induction hypothesis, application of Theorem 2 gives $F_{1} * F_{2} * \ldots * F_{k+1} \in \mathscr{S}$.

In the sequel we need some convolution closure properties for linear combinations with positive coefficients of random variables with a subexponential distribution.

In the results below we will use the notation $H_{a, b}$ for the d.f. of the random variable $a X+b Y$ and $H=H_{1,1}=F * G$.

Theorem 4. Suppose $X, Y$ are independent random variables with d.f. $F \in$ $\mathscr{S}, G \in \mathscr{L}, a, b>0$ and one of the following holds

a $\bar{G}(c x)=O(\bar{F}(x))$ for all $c>0$.

b $F, G \in \mathscr{D}$

c $F($ or $G) \in \mathscr{D}$ and $\sup _{x} \bar{G}(x) / \bar{F}(x)<\infty$.

d $\bar{F}(x) \asymp \bar{G}(x)(x \rightarrow \infty)$

Then $H_{a, b} \in \mathscr{S}$ and

$$
\bar{H}_{a, b}(x) \sim \bar{F}(x / a)+\bar{G}(x / b)(x \rightarrow \infty) .
$$

Moreover, if $F \in \mathscr{S}, a, b>0$ and $\bar{G}(c x)=o(\bar{F}(x))$ for all $c>0$, then $H_{a, b} \in \mathscr{S}$ and $H_{a, b}(x) \sim \bar{F}(x / a)$.

Proof of Theorem 4. Since a r.v. $X$ has d.f. $F \in \mathscr{S}$ if and only if the r.v. $c X$ has d.f. in $\mathscr{S}$ for some (all) $c>0$, it is sufficient to show $H_{a, b} \in \mathscr{S}$ in case $a=1$.

a In order to prove the first statement, note that as in the proof of Prop. 1 in Embrechts et al. [10], we find $\bar{H}_{1, b}(x)=O(\bar{F}(x))$ as $x \rightarrow \infty$ for $b>0$, which implies $\bar{H}_{1, b}(x) \asymp \bar{F}(x)$ (since $\bar{H}_{1, b}(x) \geq$ $\bar{F}(x)$ ). Since $\mathscr{L}$ is closed under convolution (see [11], thm. 3 ) and 
$F \in \mathscr{S} \subset \mathscr{L}$, we have $H_{1, b} \in \mathscr{L}$. Since $F, H_{1, b} \in \mathscr{L}, F \in \mathscr{S}$ and $\bar{H}_{1, b}(x) \asymp \bar{F}(x)$, application of Klüppelberg [23], Thm. 2.1 shows that $H_{1, b} \in \mathscr{S}$, hence $H_{a, b} \in \mathscr{S}$ for $a, b>0$. Observe that (since $F, G \in \mathscr{L}$ ) the d.f. of $a X \vee b Y$ is in $\mathscr{L}$, being asymptotic to $\bar{F}(x / a)+\bar{G}(x / b)$. Because $H_{a, b} \in \mathscr{S}$, application of Lemma 1 in [11] implies (2.17).

b Since $F(. / a), G(. / b) \in \mathscr{L} \cap \mathscr{D} \subset \mathscr{S}$, it follows that $F(. / a), G(. / b) \in$ $\mathscr{D} \cap \mathscr{S}$. Now [11], prop. 2 implies that $H_{a, b} \in \mathscr{S}$. Observe that once $H_{a, b} \in \mathscr{S}$ is proved, relation (2.17) follows by application of theorem 2 , since $F(. / a), G(. / b) \in \mathscr{S}$.

c Note that the assumption in $a$. is satisfied.

d Since for $a \leq 1 \sup _{x} \bar{F}(x / a)=O(\bar{G}(x))$ we can proceed as in 1 . to find $H_{a, 1} \in \mathscr{S}$ for $a \leq 1$. The proof of $H_{1, b} \in \mathscr{S}$ for $b<1$ is similar. Combination of these results then gives $H_{a, b} \in \mathscr{S}$ for $a, b>0$.

The final statement follows similarly using Proposition 1 in [10].

Corollary 2. (see Geluk [16], thm. 2) Suppose $X, Y$ are independent random variables with d.f. $F \in \mathscr{S}, G \in \mathscr{L}$ and $\bar{G}(x)=O(\bar{F}(x))$, then $H \in \mathscr{S}$ and $\bar{H}(x) \sim \bar{F}(x)+\bar{G}(x)$, hence $F \sim_{M} G$.

\section{WEIGHTED SUMS OF I.I.D. RANDOM VARIABLES IN $\mathscr{S}$}

In order to prove theorem 1 we need some auxiliary results. The first Lemma (see [16]) shows that it is sufficient to study the behaviour of nonnegative random variables under maxima and convolutions. The next Lemma is well known (see e.g. Willekens [40], Omey [31]). Observe that in this section we use the assumption that $X, Y$ be independent with d.f. $F, G$.

Lemma 3. ([16]) Let $F, G \in \mathscr{L}$. Then as $x \rightarrow \infty$

$$
P\left(X^{+} \vee Y>x\right) \sim P(X \vee Y>x) .
$$

and

$$
P\left(X^{+}+Y>x\right) \sim P(X+Y>x) .
$$

Lemma 4. ([40],[31]), $F \in \mathscr{S}$ if and only if $F^{+} \in \mathscr{S}$. Moreover if $X$ and $Y$ are r.v.'s satisfying $P(X>x) \sim P(Y>x)(x \rightarrow \infty)$, then $F \in \mathscr{S}$ if and only if $G \in \mathscr{S}$.

Lemma 5. Suppose $F \in \mathscr{S}, G \in \mathscr{L}$ and

$$
G \in \mathscr{S} \text {. }
$$

Then the following statements are equivalent. 

a $F \sim_{M} G^{+}$
b $F * G^{+} \in \mathscr{S}$
c $F \sim_{M} G$
d $F * G \in \mathscr{S}$

If (3.3) is replaced with $\bar{G}(x)=O(\bar{F}(x))(x \rightarrow \infty)$, then a-d hold.

Moreover in one (or more) of the above statements $F\left(G, G^{+}\right)$may be replaced with $F^{+}\left(G^{+}, G\right)$.

Proof of Lemma 5. Note that $F \in \mathscr{S} \subset \mathscr{L}$.

$a \Leftrightarrow b$ In view of Lemmas 3 and 4 the proof is complete by showing that $F^{+} \sim_{M} G^{+}$and $F^{+} * G^{+} \in \mathscr{S}$ are equivalent. In case $G \in \mathscr{S}$, Theorem 2 establishes this equivalence. Moreover $a \Leftrightarrow c$ and $b \Leftrightarrow d$ follow from Lemmas 3 and 4.

Under the assumption $\bar{G}(x)=O(\bar{F}(x))$ both $F^{+} \sim_{M} G^{+}$and $F^{+} * G^{+} \in$ $\mathscr{S}$ follow from Corollary 2.

The final statement follows from Lemmas 3 and 4.

Lemma 6. Let $X, Y$ be independent, $F \in \mathscr{S}, G^{-} \in \mathscr{L}$ and $P(Y<$ $-c x)=O(\bar{F}(x))$ for $c>0$ as $x \rightarrow \infty$.

Then $H_{a,-b} \in \mathscr{S}$ for $a, b>0$ and

$\bar{H}_{a,-b}(x)=P(a X-b Y>x) \sim P(X>x / a)+P(Y<-x / b)(x \rightarrow \infty)$.

In particular, under the assumptions of Theorem 1, if $\vee_{1}^{m} c_{i}>0$, it follows that

$$
P\left(\sum_{i=1}^{m} c_{i} X_{i}>x\right) \sim \sum_{i=1}^{m} P\left(c_{i} X_{i}>x\right)(x \rightarrow \infty) .
$$

Proof of Lemma 6. Under condition bl(b3) in Theorem 1, the Lemma follows immediately from Theorem $4 \mathrm{a}(4 \mathrm{~d})$ and Lemma 5 . If condition $b 2$ is satisfied, the final statement in Theorem 4 completes the proof.

Lemma 7. Let $X_{n}, Y_{n}(n=1,2, \ldots)$ be independent with d.f.'s $F_{n}, G_{n}$ respectively, $F_{n} \in \mathscr{S}(n=1,2, \ldots)$ and for $\delta>0$, there exist $x_{n}^{\prime}=$ $x_{n}^{\prime}(\delta), n=1,2, \ldots$ such that for $x>x_{n}^{\prime}, P\left(Y_{n}>x\right) \leq \delta P\left(X_{n}>x\right), n=$ $1,2, \ldots$. Then for $\varepsilon>0$ there exist $x_{n}=x_{n}(\varepsilon)$ such that for $x>x_{n}, n \geq 1$

$$
(1-\varepsilon) P\left(X_{n}>x\right) \leq P\left(X_{n}+Y_{n}>x\right) \leq(1+\varepsilon) P\left(X_{n}>x\right) .
$$

Proof of Lemma 7. Take $\varepsilon \in(0,1)$. Note that for $x>x^{*}(n)$ and $n=$ $1,2, \ldots$

$$
P\left(X_{n}+Y_{n}>x\right) \leq P\left(X_{n}^{+}+Y_{n}^{+}>x\right) \leq(1+\varepsilon) P\left(X_{n}>x\right),
$$

the proof of the last inequality being similar to that of Proposition 1 in [10]. Take $a_{n}$ such that $P\left(Y_{n}>a_{n}\right)<\varepsilon$ for all $n \geq 1$. Since $F_{n} \in \mathscr{S} \subset \mathscr{L}$, 
application of Fatou's lemma gives

$$
\begin{gathered}
\liminf _{x \rightarrow \infty} \frac{P\left(X_{n}+Y_{n}>x\right)}{P\left(X_{n}>x\right)} \geq \liminf _{x \rightarrow \infty} \frac{P\left(X_{n}+Y_{n}>x, Y_{n} \leq a_{n}\right)}{P\left(X_{n}>x\right)} \geq \\
\int_{-\infty}^{a_{n}} \liminf _{x \rightarrow \infty} \frac{\bar{F}_{n}(x-y)}{\bar{F}_{n}(x)} d G_{n}(y)=P\left(Y_{n} \leq a_{n}\right)>1-\varepsilon .
\end{gathered}
$$

Now (3.6) follows by letting $\varepsilon \downarrow 0$.

Lemma 8. Under the conditions of theorem 1, for $\varepsilon>0$ there exists $m_{0}$ such that

$P\left(\sum_{j>m}\left|c_{j} X_{j}\right|>x\right) \leq(1+\varepsilon) \sum_{j>m} P\left(\left|c_{j} X_{j}\right|>x\right)$ for $m>m_{0}, x>x_{0}(\varepsilon)$.

Proof of Lemma 8. First assume $0<\beta<1$ in (1.5). As in the proof on $\mathrm{p}$. 228 of Resnick [35], it is sufficient to show that

$$
P\left(\sum_{j>m}\left|c_{j} X_{j}\right| \mathbf{1}_{\left|c_{j} X_{j}\right| \leq x}>x\right)=O(P(|X|>x))(x \rightarrow \infty) .
$$

Writing $K$ for the distribution function of $|X|$ and $\bar{K}=1-K$, for $\varepsilon>0$ arbitrary we have

$$
(1-\varepsilon) s^{-\beta} \leq \bar{K}(x s) / \bar{K}(x) \leq(1+\varepsilon) s^{-\alpha} \text { for } s \geq 1, x>x_{0}
$$

(see Bingham et al. [2], Ch. 2 on extended regular variation). Since $\beta<1$, we have $x \bar{K}(x) \rightarrow \infty$ as $x \rightarrow \infty$ and it follows that for $x$ sufficiently large

$$
\begin{aligned}
\frac{E\left(\left|X_{j}\right| \mathbf{1}_{\left|X_{j}\right| \leq x}\right)}{x \bar{K}(x)} & =\frac{\int_{0}^{x} s d K(s)}{x \bar{K}(x)}=\int_{0}^{x_{0} / x}+\int_{x_{0} / x}^{1} \frac{\bar{K}(x s)}{\bar{K}(x)} d s-1 \\
& \leq \varepsilon+\frac{1}{1-\varepsilon} \int_{0}^{1} s^{-\beta} d s-1 \\
& \leq \frac{2 \varepsilon+\beta}{(1-\varepsilon)(1-\beta)} .
\end{aligned}
$$

As a consequence, it follows that for $m$ such that $\vee_{j \geq m} c_{j}<1$ and $x$ sufficiently large

$$
\begin{aligned}
\frac{P\left(\sum_{j>m}\left|c_{j} X_{j}\right| \mathbf{1}_{\left|c_{j} X_{j}\right| \leq x}>x\right)}{P(|X|>x)} & \leq \frac{\sum_{j>m}\left|c_{j}\right| E\left|X_{j}\right| \mathbf{1}_{\left|X_{j}\right| \leq x\left|c_{j}^{-1}\right|}}{x P(|X|>x)} \\
& =\sum_{j>m} \frac{\left|c_{j}\right| \int_{0}^{x /\left|c_{j}\right|} s d K(s)}{x \bar{K}\left(x /\left|c_{j}\right|\right)} \cdot \frac{\frac{x}{\left|c_{j}\right|} \bar{K}\left(\frac{x}{\left|c_{j}\right|}\right)}{x \bar{K}(x)} \\
& \leq(1+\varepsilon) \frac{2 \varepsilon+\beta}{(1-\varepsilon)(1-\beta)} \sum_{j>m}\left|c_{j}\right|^{\alpha}<\infty .
\end{aligned}
$$


In case $\beta \geq 1$ in (1.5), an inductive procedure as in Resnick [35] or Mikosch and Samorodnitsky [30] completes the proof.

Proof of Theorem 1. Note that without loss of generality we may assume that $c_{1}=1$. By assumption there exists $d$ such that

$$
P(X<-x) \leq d P(X>x) \text { for } x>0 .
$$

Take $\varepsilon>0$. Write $Z=\sum c_{i} X_{i}=\sum_{i \leq m}+\sum_{i>m}=: Z_{m}+Z_{m}^{\prime}$, where $m$ is such that $\left|c_{i}\right|<1$ for $i>m$.

Now condition (3.7) ensures that for $x$ sufficiently large

$$
\sum_{i>m} P\left(\left|c_{i} X_{i}\right|>x\right) \leq(1+\varepsilon) \sum_{i>m}\left|c_{i}\right|^{\alpha} P(|X|>x) .
$$

Hence in view of Lemma 8, (3.7) and (3.8), we have

$$
\begin{aligned}
P\left(Z_{m}^{\prime}>x\right) & \leq P\left(\left|Z_{m}^{\prime}\right|>x\right) \\
& \leq P\left(\sum_{i>m}\left|c_{i} X_{i}\right|>x\right) \\
& \leq(1+\varepsilon) \sum_{i>m} P\left(\left|c_{i} X_{i}\right|>x\right) \\
& \leq(1+\varepsilon)^{2}(1+d) \sum_{i>m}\left|c_{i}\right|^{\alpha} P(X>x) .
\end{aligned}
$$

From (3.5) it follows that for $x$ sufficiently large

$$
P\left(Z_{m}>x\right) \geq(1-\varepsilon) \sum_{1}^{m} P\left(c_{i} X_{i}>x\right) \geq(1-\varepsilon) P(X>x) .
$$

Combination with (3.10) then gives

$$
P\left(Z_{m}^{\prime}>x\right) \leq(1-\varepsilon)^{-1}(1+\varepsilon)^{2}(1+d) \sum_{i>m}\left|c_{i}\right|^{\alpha} P\left(Z_{m}>x\right) \text { for } x>x(\varepsilon) \text {. }
$$

Application of Lemma 7 (note that the d.f. of $Z_{m}$ is subexponential by Cor. 1) and (3.5) shows that for $\varepsilon>0$ there exists $m=m(\varepsilon)$ such that for $x>x_{1}(m, \varepsilon)$

$$
\begin{aligned}
P(Z>x) & <(1+\varepsilon) P\left(Z_{m}>x\right) \\
& \leq(1+\varepsilon)^{2} \sum_{i \leq m} P\left(c_{i} X_{i}>x\right) \\
& \leq(1+\varepsilon)^{2} \sum_{i} P\left(c_{i} X_{i}>x\right) .
\end{aligned}
$$

A lower inequality is obtained as follows. Since $c_{1}=1$, using (3.8) and (3.9) it follows that

$$
\frac{\sum_{i>m} P\left(c_{i} X_{i}>x\right)}{\sum_{i \leq m} P\left(c_{i} X_{i}>x\right)} \leq \frac{\sum_{i>m} P\left(\left|c_{i} X_{i}\right|>x\right)}{P(X>x)} \leq(1+\varepsilon)(1+d) \sum_{i>m}\left|c_{i}\right|^{\alpha} .
$$

Similar to (3.13) we then find that for $x>x(\varepsilon)$ 


$$
\begin{aligned}
P(Z>x) & >(1-\varepsilon) P\left(Z_{m}>x\right) \geq(1-\varepsilon)^{2} \sum_{i \leq m} P\left(c_{i} X_{i}>x\right) \\
& \geq(1-\varepsilon)^{3} \sum_{i} P\left(c_{i} X_{i}>x\right) .
\end{aligned}
$$

Since $\varepsilon>0$ is arbitrary, the statement of the theorem now follows from (3.13) and (3.14).

\section{EQUITY RETURNS OF REINSURERS}

In the introduction we argued that both sides of the balance sheets of insurance companies consist in linear combinations of risk factors also held by other reinsurers. We now express the two asset sides of the balance sheet into a mathematical format and analyze what this implies for the distribution of a reinsurer's equity.

4.1. asset side; the investment portfolio. Suppose there are two reinsurers who allocate their capital and invest premium income in just two independent assets. For simplicity, let the first asset be a zero coupon bond with return $X$, while the second asset is a non-dividend paying share with return $Y$. Let reinsurer one hold the portfolio $Q=(1-\gamma) X+\gamma Y$, while the investment portfolio of reinsurer two is $W=\gamma X+(1-\gamma) Y$ where $\gamma$ is restricted to be in the interval between zero and one, i.e. there are no short sales. We assume that $X$ and $Y$ are independent with subexponential distributions and that (one of) the assumptions of Theorem 4 is satisfied. It follows that the portfolios $Q$ and $W$ each have a subexponential distribution.

4.2. affine claim models; liability side. To model the returns on the liability side we consider treaties under the quota-share contract, which are written on two underlying risks which are assumed to be independent. Each company writes reinsurance treaties on both risks. Notice that since this is in the proportional business, both reinsurers share proportionally in the risks they reinsure. Suppose these proportions are indicated by $\lambda$. Let $a$ and $b$ be the premium incomes per unit investment. The premium incomes are assumed to be non-random. In line with the empirical evidence we assume that claim sizes per unit investment follow subexponential distributions. Let the respective claim sizes be $A$ and $B$ respectively. Whether a claim materializes is modelled using a simple Bernoulli distribution. It is common to model the claim arrival process as a compound Poisson process. Since we work in a atemporal setting, a simple Bernoulli distribution can capture this feature. Let the two i.i.d. Bernoulli random variables be $N$ and $M$ respectively where $\pi=P(N=1)=1-P(N=0)$ and a similar expression 
for $M$. The liability sides (including positive premium income) of the two reinsurers now looks as follows

$$
K=(1-\lambda) a+\lambda b-N(1-\lambda) A-M \lambda B
$$

and

$$
L=\lambda a+(1-\lambda) b-N \lambda A-M(1-\lambda) B .
$$

Assuming that $A$ and $B$ have independent subexponential distribution functions and satisfy one of the assumptions of Theorem 4, it follows immediately that $K$ and $L$ are subexponentially distributed (in the left tail). Thus in case both sides of the balance sheet have independent subexponential distributions, the results in section 3 provide us with a tool to analyze this situation.

Many reinsurance contracts do not have unlimited claim sizes, but are capped. Suppose we capture this by assuming that the distribution of a capped claim is light tailed. We consider the mixed case where some claims are capped and others are not. Specifically assume that $A$ is subexponential, while $B$ is light tailed. In that case, the next Lemma shows that the sum of the light and the heavy tail leads naturally to subexponentiality.

Lemma 9. Suppose $A, B$ are independent random variables with d.f. $F, G$ and $H$ is the d.f. of the convolution $A+B$. If $F \in \mathscr{S}$ and $\bar{G}(x)=o(\bar{F}(x))$ as $x \rightarrow \infty$, then $H \in \mathscr{S}$ and $\bar{H}(x) \sim \bar{F}(x)$.

Proof of Lemma 9. The statement of the Lemma is the same as in Prop. 1a of [10], without the assumption that the random variables are a.s. nonnegative. Application of this result shows that

$P(A+B>x) \leq P\left(A^{+}+B^{+}>x\right) \sim P\left(A^{+}>x\right)=P(A>x)(x \rightarrow \infty)$.

Conversely, for $\varepsilon \in(0,1)$ arbitrary, choose $a$ such that $\bar{G}(a)=P(B>$ a) $>1-\varepsilon$. Then for $x$ sufficiently large

$$
\begin{aligned}
\frac{H(x)}{\bar{F}(x)} & =\int_{-\infty}^{\infty} \frac{\bar{G}(x-y)}{\bar{F}(x)} d F(y) \geq \int_{x-a}^{\infty} \frac{\bar{G}(x-y)}{\bar{F}(x)} d F(y) \\
& \geq(1-\varepsilon) \frac{\bar{F}(x-a)}{\bar{F}(x)} \geq 1-2 \varepsilon
\end{aligned}
$$

since $F \in \mathscr{S} \subset \mathscr{L}$. Combination of the estimates shows that $\bar{H}(x) \sim$ $\bar{F}(x)$, whence $H \in \mathscr{S}$, which completes the proof.

In the next section we investigate the joint behavior of the total net returns. 


\section{CO-CRASH PROBABILITIES}

In order to describe dependence of markets, the standard correlation measure has the advantages of being easy to calculate, it is well behaved under linear operations and it is the natural measure in case of normally distributed random variables. In case of heavy tailed random variables, apart from the fact that the coefficient of correlation may be undefined, its main disadvantage is that it does not capture very well possible dependence in the tails. What worries supervisors and industry representatives is that a heavy loss in one market might go hand in hand with a heavy loss in another market, destroying the real value of a diversified investment portfolio. We are looking for a measure which captures the potential for systemic breakdown. Consider the following example. Suppose two reinsurers have risk profiles $S$ and $T$, where $S$ and $T$ reflect the composite asset and liability sides of the reinsurers, e.g. $S=Q+K$ and $T=W+L$ as discussed in the previous section.

Naturally one asks given that $T>t$, what is the probability that $S>s$, where $t, s$ are high loss levels. For notational simplicity, we take the two levels on which we condition equal to $s$, but this is by no means necessary, as is indicated in Theorem 5 below. If we assume that the marginal distributions are equal (which we do in the definition below), a natural definition of asymptotic independence is

$$
P(S>s \mid T>s) \rightarrow 0 \text { as } s \rightarrow \infty .
$$

Note that equality of the marginal distributions can be obtained using a suitable transformation on $S$ and/or $T$.

Instead of this conditional probability, we will adopt the related probability that conditions on any company's crash, without indicating the specific reinsurer. This is the linkage measure

$$
\kappa_{s}:=\frac{P\{S>s\}+P\{T>s\}}{1-P\{S \leq s, T \leq s\}}
$$

proposed in Embrechts et al.[14]. The linkage measure $\kappa_{s}$ reflects the expected number $E\left\{N_{s} \mid N_{s} \geq 1\right\}$ of reinsurer's crashes $N_{s}$, given that least one reinsurer has collapsed, i.e. given $S \vee T>s$.

Alternatively one may write

$$
\kappa_{s}=E\left\{N_{s} \mid N_{s} \geq 1\right\}=1+\frac{P(S \wedge T>s)}{P(S \vee T>s)} .
$$

Since $P(S \vee T>s)=2 P(S>s)-P(S \wedge T>s)$, it follows that (5.1) is equivalent to $P(S \vee T>s) \sim 2 P(S>s)$, or equivalently

$$
\frac{P(S \wedge T>s)}{P(S \vee T>s)} \sim \frac{1}{2} P(S>s \mid T>t) .
$$


As a consequence, asymptotic independence is equivalent to the statement $\kappa_{s} \rightarrow 1$ as $s \rightarrow \infty$. We prefer the expression $E\left\{N_{s} \mid N_{s} \geq 1\right\}$ since it can naturally be generalized to higher dimensions.

An open issue is on which level of $s$ one speaks of a crash or company default. Economics does not provide a natural default level, such as is the case when we think of a flood in terms of the dike height. Crash levels differ per asset category and circumstances. For this reason we are interested in the asymptotic behavior of $\kappa_{s}$ as $s \rightarrow \infty$.

Obviously $1 \leq \kappa_{s} \leq 2$. Consider two extreme cases.

- If $S$ and $T$ are independent with d.f.'s $F$ and $G$, we have $\kappa_{s}=$ $1+\bar{F}(s) \bar{G}(s) /(\bar{F}(s)+\bar{G}(s)-\bar{F}(s) \bar{G}(s))$, hence the rate at which $\kappa_{s}$ approaches 1 as $s \rightarrow \infty$ is given by $\kappa_{s}-1 \sim \bar{F}(s) \bar{G}(s) /(\bar{F}(s)+$ $\bar{G}(s))$.

- If $P(X=Y)=1$, obviously $\kappa_{s}=2$.

It should be observed that $\kappa=\lim _{s \rightarrow \infty} \kappa_{s}$ may be equal to 1 even if the components are dependent. This is typical for the situation of light tails, like the bivariate normal distribution.

In what follows we investigate the interdependency between two reinsurer's asset or liability sides, cf. section 4. Using similar methods, these results can be extended to linear combinations of more than two random variables, such as in the case of combining the asset and liability sides. Using the linear framework for balance sheet items as explained in section 4, we consider the total risks $S=a_{1} X+b_{1} Y$ and $T=a_{2} X+b_{2} Y$ of two markets which both have standard normal distributions. Then Sibuya's result [37] shows that the total risks are asymptotically independent in the sense of (5.1). See also Resnick [35], Proposition 5.27.

Since the risk profiles of stocks are often heavy tailed, we are interested in the case of subexponential tail behavior. In that situation, asymptotic dependence is to be expected. Before we discuss general portfolios, we give a simple example where this occurs.

Proposition 1. [17] Consider the risk profiles of two portfolios $X+Z$ and $Y+Z$, where $X, Y$ and $Z$ are i.i.d. with subexponential distribution function. Then $\kappa=\lim _{s \rightarrow \infty} \kappa_{s}=4 / 3$.

For two linear portfolios with independent loss levels $X, Y$ having subexponential distributions, satisfying $H_{a, b} \in \mathscr{S}$ for $a, b>0\left(H_{a, b}\right.$ is the d.f. of $a X+b Y)$, Theorem 5 below implies that there can be asymptotic dependence and

$$
\kappa_{s}-1 \sim \frac{\bar{F}\left(\left(a_{1}^{-1} \vee a_{2}^{-1}\right) s\right)+\bar{G}\left(\left(b_{1}^{-1} \vee b_{2}^{-1}\right) s\right)}{\bar{F}\left(\left(a_{1}^{-1} \wedge a_{2}^{-1}\right) s\right)+\bar{G}\left(\left(b_{1}^{-1} \wedge b_{2}^{-1}\right) s\right)}
$$


If $X, Y$ are i.i.d. with regularly varying tail functions, it follows that the limiting expected additional number of crashes as $s \rightarrow \infty$, given at least one crash at level $k$, is $\kappa-1=\lim _{s \rightarrow \infty} \kappa_{s}-1$ and the limit exists in the interval $(1,2]$. Similarly we find in this case that $\kappa=1$ for subexponential d.f.'s with a rapidly varying tail, unless $a_{1}=a_{2}$ and $b_{1}=b_{2}$.

This observation underlines the phenomenon that the heavy tailed behavior of asset returns may lead to increased risk, even for diversified portfolios.

For reasons of notational simplicity we formulate the result below for two random variables.

Theorem 5. Suppose $X, Y$ are independent random variables with d.f. $F, G \in \mathscr{S}$ and $Z=a X+b Y$ has d.f. $H_{a, b} \in \mathscr{S}$ for $a, b>0$.

Suppose $\lambda_{1}, \lambda_{2}, \mu_{1}, \mu_{2}, x, y>0$ and both coordinates of the point of intersection $S$ of the lines $\lambda_{1} X+\lambda_{2} Y=t$ and $\mu_{1} X+\mu_{2} Y=t$ are positive for $t>0$. Then as $t \rightarrow \infty$

$P\left(\lambda_{1} X+\lambda_{2} Y>t x \cup \mu_{1} X+\mu_{2} Y>t y\right) \sim \bar{F}\left(\left(\frac{x}{\lambda_{1}} \wedge \frac{y}{\mu_{1}}\right) t\right)+\bar{G}\left(\left(\frac{x}{\lambda_{2}} \wedge \frac{y}{\mu_{2}}\right) t\right)$.

Corollary 3. Under the conditions of Theorem 5 it follows that

$P\left(\lambda_{1} X+\lambda_{2} Y>t x \cap \mu_{1} X+\mu_{2} Y>t y\right) \sim \bar{F}\left(\left(\frac{x}{\lambda_{1}} \vee \frac{y}{\mu_{1}}\right) t\right)+\bar{G}\left(\left(\frac{x}{\lambda_{2}} \vee \frac{y}{\mu_{2}}\right) t\right)$.

The special case of regularly varying tail functions leads to the following Corollary. We only formulate the result corresponding to Theorem 5, the analogue of Corollary 3 being similar.

Corollary 4. Suppose $X_{1}, X_{2}$ are i.i.d. with distribution function $F$ with a regularly varying tail function $\bar{F}$ with index $-\alpha$. Then (with $\lambda_{i}, \mu_{i}, x, y$ as above) as $t \rightarrow \infty$

$$
\begin{aligned}
& P\left(\lambda_{1} X_{1}+\lambda_{2} X_{2}>t x\right.\left.\cup \mu_{1} X_{1}+\mu_{2} X_{2}>t y\right) \sim \\
&\left\{\left(\frac{x}{\lambda_{1}} \wedge \frac{y}{\mu_{1}}\right)^{-\alpha}+\left(\frac{x}{\lambda_{2}} \wedge \frac{y}{\mu_{2}}\right)^{-\alpha}\right\} P\left(X_{1}>t\right) .
\end{aligned}
$$

In order to prove the theorem, we need a simple Lemma.

Lemma 10. Suppose $X, Y$ are independent r.v's with distribution functions $F, G \in \mathscr{S}, F \sim_{M} G$ and $a, b>0$ are constants. Then

$$
\bar{F}(a t) \bar{G}(b t)=o\{\bar{F}((a+b) t)+\bar{G}((a+b) t)\},(t \rightarrow \infty) .
$$

Proof of Lemma 10. Fix $\varepsilon \in(0,1)$. Then

$$
\begin{aligned}
P(X>\varepsilon t & Y>(1-\varepsilon) t) \\
& =P(X>t, Y>(1-\varepsilon) t)+P(\varepsilon t<X \leq t, Y>t) \\
& +P(\varepsilon t<X \leq t, \underset{22}{(1-\varepsilon) t<Y \leq t)=: A+B+C .}
\end{aligned}
$$


Then $A=\bar{F}(t) \bar{G}((1-\varepsilon) t)=o(\bar{F}(t))$ and $B=(\bar{F}(\varepsilon t)-\bar{F}(t))) \bar{G}(t)=$ $o(\bar{G}(t))$. Moreover, since $F \sim_{M} G$, it follows that $C \leq P(X+Y>$ $t)-P(X \vee Y>t)=o(\bar{F}(t)+\bar{G}(t))$.

Combination of the estimates and replacing $\varepsilon$ with $a /(a+b)$ and $t$ with $(a+b) t$ completes the proof.

Proof of Theorem 5. The case in which the random variables take negative values with non-zero probability can be reduced to the non-negative case by Lemmas 3 and 4, so we assume that $X, Y>0$ with probability 1 .

Since $H_{a, b} \in \mathscr{S}$ for $a, b>0$, using Lemma 5, it follows that $F(. / a) \sim_{M}$ $G(. / b)$, hence

$$
\begin{aligned}
& P\left(\lambda_{1} X+\lambda_{2} Y>t, \lambda_{1} X \vee \lambda_{2} Y \leq t\right) \\
& \quad=P\left(\lambda_{1} X+\lambda_{2} Y>t\right)-P\left(\lambda_{1} X \vee \lambda_{2} Y>t\right)=o\left(\bar{F}\left(t / \lambda_{1}\right)+\bar{G}\left(t / \lambda_{2}\right)\right) .
\end{aligned}
$$

Observe that it is sufficient to prove the theorem for $x=y=1$ (replace $\lambda_{i}, \mu_{i}$ with $\left.\lambda_{i} x, \mu_{i} y\right)$.

Write $p:=P\left(\lambda_{1} X+\lambda_{2} Y>t \cup \mu_{1} X+\mu_{2} Y>t\right)$ and $\left(t_{1}, t_{2}\right)=$ $\left(\frac{\lambda_{2}-\mu_{2}}{\lambda_{2} \mu_{1}-\lambda_{1} \mu_{2}} t, \frac{\mu_{1}-\lambda_{1}}{\lambda_{2} \mu_{1}-\lambda_{1} \mu_{2}} t\right)$ for the coordinates of the point S. Suppose

$$
\mu_{2}<\lambda_{1}<\mu_{1}<\lambda_{2} .
$$

We split the required probability into three parts.

$$
\begin{gathered}
p=P\left(\lambda_{1} X+\lambda_{2} Y>t, X \leq t_{1}\right)+P\left(\mu_{1} X+\mu_{2} Y>t, Y \leq t_{2}\right)+ \\
P\left(X>t_{1}, Y>t_{2}\right)=: I+I I+I I I .
\end{gathered}
$$

Since $t_{1} \rightarrow+\infty$ as $t \rightarrow \infty, I \geq \bar{G}\left(t / \lambda_{2}\right) F\left(t_{1}\right) \sim \bar{G}\left(t / \lambda_{2}\right)$.

Moreover $I \leq \bar{G}\left(t / \lambda_{2}\right) F\left(t_{1}\right)+P\left(\lambda_{1} X+\lambda_{2} Y>t, \lambda_{1} X \vee \lambda_{2} Y \leq t\right)$.

Combination of the lower and upper estimates with (5.5) shows that

$I=\bar{G}\left(t / \lambda_{2}\right)+o\left(\bar{F}\left(t / \lambda_{1}\right)+\bar{G}\left(t / \lambda_{2}\right)\right)=\bar{G}\left(t / \lambda_{2}\right)+o\left(\bar{F}\left(t / \mu_{1}\right)+\bar{G}\left(t / \lambda_{2}\right)\right)$.

Similarly we find $I I=\bar{F}\left(t / \mu_{1}\right)+o\left(\bar{F}\left(t / \mu_{1}\right)+\bar{G}\left(t / \mu_{2}\right)\right)=\bar{F}\left(t / \mu_{1}\right)+$ $o\left(\bar{F}\left(t / \mu_{1}\right)+\bar{G}\left(t / \lambda_{2}\right)\right)$.

The last term can be estimated as follows. From (5.6) it follows that $t_{1}+t_{2}>t / \mu_{1} \vee t / \lambda_{2}=t / \mu_{1}$.

As a consequence, for $t \rightarrow \infty$, by Lemma 8,

$I I I=\bar{F}\left(t_{1}\right) \bar{G}\left(t_{2}\right)=o\left(\bar{F}\left(t_{1}+t_{2}\right)+\bar{G}\left(t_{1}+t_{2}\right)\right)=o\left(\bar{F}\left(t / \mu_{1}\right)+\bar{G}\left(t / \lambda_{2}\right)\right)$,

hence combination of the estimates gives $p \sim \bar{F}\left(t / \mu_{1}\right)+\bar{G}\left(t / \lambda_{2}\right)$.

The other cases $\left(\lambda_{1}<\mu_{2}<\lambda_{2}<\mu_{1}, \mu_{1}<\lambda_{2}<\mu_{2}<\lambda_{1}\right.$ and $\lambda_{2}<$ $\left.\mu_{1}<\lambda_{1}<\mu_{2}\right)$ can be proved similarly.

Proof of Corollary 3. This follows trivially from theorem 5. 
Proof of Corollary 4. Note that random variables with a regularly varying tail function are subexponential. Corollary 1 together with Lemmas 3 and 4 now ensure that the condition $H_{a, b} \in \mathscr{S}$ is satisfied. Conclusion (5.4) now follows from (5.2), using the regular variation of $\bar{F}$.

\section{REFERENCES}

[1] Asmussen, S. (2000). Ruin probabilities. World Scientific Publishing Co., Inc., River Edge, NJ.

[2] Bingham, N., Goldie, C. and Teugels, J. (1987). Regular Variation. Cambridge University Press.

[3] Blattberg, R.C. and N.J. Gonedes (1974), 'A Comparison of the Stable and Student Distributions as Statistical Models for Stock Prices', Journal of Business, 47, 244280.

[4] Chistyakov, V.P., (1964). A theorem on sums of independent, positive random variables and its applications to branching processes, Theory Prob. Appl. 9, 640-648.

[5] Cline, D.B.H., (1983). Estimation and linear prediction for regression, autoregression and ARMA with infinite variance data, Ph. D. Thesis, Dept. of Statistics, Colorado State University, Fort Collins, CO80523 USA.

[6] Cline, D.B.H., (1986). Convolution tails, product tails and domains of attraction, Prob. Th. Rel. Fields 72, 529-557.

[7] Cline, D.B.H., Hsing, T, (1989), Large deviation probabilities for sums of random variables with heavy or subexponential tails, preprint Texas A\&M University, preprint.

[8] Danielsson, J. and C.G. de Vries (1997), 'Tail index and quantile estimation with very high frequency data', Journal of Empirical Finance, 4, 241-257.

[9] Davis, R.A., Resnick, S. (1988). Extremes of moving averages of random variables from the domain of attraction of the double exponential distribution, Stoc. Proc. Appl. 30, 41-68.

[10] Embrechts, P., Goldie, C., Veraverbeke, N. (1979). Subexponentiality and infinite divisibility, Z. Wahrscheinlichkeitstheorie, 49, 335-347.

[11] Embrechts, P., Goldie, C., (1980). On closure and factorization properties of subexponential and related distributions, J. Austr. Math. Soc. 29, 243-256.

[12] Embrechts, P., Goldie, C., (1982). On convolution tails, Stoch. Proc. Appl. 13, 263278.

[13] Embrechts, P., Klüppelberg, C., Mikosch, T. (1997). Modelling Extremal Events, Springer Verlag.

[14] Embrechts, P., de Haan, L., Xin Huang (2000). Modelling multivariate extremes, pp. 59-67 in Extremes and integrated risk management, UBS Warburg

[15] Feller, W. (1971). An Introduction to Probability Theory and Its Applications. Volume II, John Wiley \& Sons, Inc.

[16] Geluk, J.L., Asymptotics in the symmetrization inequality, Stat. Prob. Letters, 69, 63-68, (2004).

[17] Geluk, J.L., de Haan, L., de Vries, C.G., (2004). Weak and strong financial fragility, in preparation.

[18] Goldie, C., (1978). Subexponential distributions and dominated variation tails, J. Appl. Prob. 15, 440-442. 
[19] de Haan, L., Jansen, D.W., Koedijk, K., and de Vries, C.G. (1994). Safety first portfolio selection, extreme value theory and long run asset risks. Extreme value theory and applications (J. Galambos et al., eds.) 471-487. Kluwer, Dordrecht.

[20] Hartmann, P., Straetmans, S. and de Vries, C.G. (2004), Asset market linkages in crisis periods, Review of Economics and Statistics, 81, 313-326.

[21] Jansen, D.W. and de Vries, C.G. (1991), 'On the frequency of large stock returns: putting booms and busts into perspective', Review of Economics and Statistics, 73,19-24.

[22] Jondeau, E. and Rockinger, M. (2003), 'Testing for differences in the tails of stockmarket returns ', Journal of Empirical Finance, 10, 559-581.

[23] Klüppelberg, C., (1988). Subexponential distributions and integrated tails. J. Appl. Prob. 25, 132-141.

[24] Kokoszka, P.S., Taqqu, M.S., (1996). Parameter estimation for infinite variance fractional ARIMA, Ann. Stat. 24, 1880-1913.

[25] Leslie, J.R., (1989). On the nonclosure under convolution of the subexponential family, J. Appl. Prob., 26, 58-66.

[26] Longin, F.M. (1996), 'The asymptotic Distribution of Extreme stock market returns', Journal of Business, 69, 383-408.

[27] Longin, F. and Solnik, B. (2001), 'Extreme correlation of international equity markets', Journal of Finance, 56, 649-676. .

[28] Lux, T. (1996), 'The stable Paretian Hypothesis and the frequency of large returns: an dexamination of major German stocks', Applied Financial Economics, 6, 463-475.

[29] Mandelbrot, B. (1963), 'The variation of certain speculative prices', Journal of Business, 36, 394-419.

[30] Mikosch, T., Samorodnitsky, G., (2000). The supremum of a negative drift random walk with dependent heavy tailed steps, Ann. Appl. Prob., 10, 1025-1064.

[31] Omey, E., (2002). On the difference between the distribution function of the sum and the maximum of real random variables, Publ. Inst. Math., 71, 63-77.

[32] Pfeifer, D., (1997). A statistical model to analyse natural catastrophe claims by means of record values, Proceedings of the 28th International Astin Colloquium.

[33] Poon, S.H., Rockinger, M. and Tawn, J. (2001), 'New extreme-value dependence measures and finance applications', CEPR Discussion Paper, no. 2762, London, April.

[34] Reiss, R.D., Thomas, M., (2001), Statistical analysis of extreme values. From insurance, finance, hydrology and other fields. Birkhäuser, Basel.

[35] Resnick, S., (1987). Extreme values, regular variation, and point processes, Springer Verlag.

[36] Rolski, T., Schlegel, S., Schmidt, V., Asymptotics of Palm-stationary buffer content distribution in fluid flow queues, Adv. Appl. Prob., 31 (1999), 235-253.

[37] Sibuya, M. (1960), Bivariate extreme statistics. I. Ann. Inst. Statist. Math. Tokyo 11, 195-210.

[38] Starica, C. (1999), 'Multivariate extremes for models with constant conditional correlations', Journal of Empirical Finance, 6, 515-553.

[39] Straetmans, S. (2000), Extremal spill-overs in equity markets, in P. Embrechts (ed.), Extremes and Integrated Risk Management, Risk Books (London), 187-204.

[40] Willekens, E., (1986). Hogere orde theorie voor subexponentiële verdelingen, Ph.D. Thesis, University of Leuven. 